\title{
MinScIE: Citation-centered Open Information Extraction
}

\author{
Anne Lauscher ${ }^{*}$ \\ University of Mannheim \\ Mannheim, Germany \\ anne@informatik.uni-mannheim.de
}

\author{
Yide Song* \\ University of Mannheim \\ Mannheim, Germany \\ yisong@mail.uni-mannheim.de
}

\author{
Kiril Gashteovski ${ }^{*}$ \\ University of Mannheim \\ Mannheim, Germany \\ k.gashteovski@uni-mannheim.com
}

\begin{abstract}
Acknowledging the importance of citations in scientific literature, in this work we present MinScIE, an Open Information Extraction system which provides structured knowledge enriched with semantic information about citations. By comparing our system to it's original core, MinIE, we show that our approach improves extraction precision by 3 percentage points.
\end{abstract}

\section{CCS CONCEPTS}

- Computing methodologies $\rightarrow$ Information extraction; Supervised learning by classification; Discourse, dialogue and pragmatics; • Applied computing $\rightarrow$ Digital libraries and archives

\section{KEYWORDS}

open information extraction, citation analysis, citation polarity, citation purpose, suppport vector machines, scitorics

\section{ACM Reference Format:}

Anne Lauscher, Yide Song, and Kiril Gashteovski. 2019. MinScIE: Citationcentered Open Information Extraction. In $\mathcal{} C D L$ '19: ACM/IEEE foint Conference on Digital Libraries, June 2-6, 2019, Urbana-Champaign, Illinois. ACM, New York, NY, USA, 2 pages.

\section{INTRODUCTION}

With the growing amount of scientific literature published per year, the automatic processing of scientific writing becomes more and more important. For the purpose of generating structured knowledge from large scientific text collections, Open Information Extraction (OIE) can be applied. OIE systems aim to extract information in the form of triples - (subject, relation, object) - from natural language sentences in an unsupervised manner. Consider the sentence "Bell is a telecommunication company, which is based in L.A." - most of the OIE systems would extract the triples ("Bell"; "is"; "telecommunication company") and ("Bell"; "is based in"; "L.A."). However, Groth et al. [4] recently showed in a systematic evaluation that current off-the-shelf OIE systems perform significantly worse when applied to the science domain, which raises the need for domain adaptation. Acknowledging the importance of citations as argumentative tools in scientific writing [3], in this work we present MinScIE, ${ }^{1}$

\footnotetext{
*All authors contributed equally to this research.

${ }^{1}$ Pronounciation: [m'mski].

Permission to make digital or hard copies of all or part of this work for personal or classroom use is granted without fee provided that copies are not made or distributed for profit or commercial advantage and that copies bear this notice and the full citation on the first page. Copyrights for components of this work owned by others than ACM must be honored. Abstracting with credit is permitted. To copy otherwise, or republish, to post on servers or to redistribute to lists, requires prior specific permission and/or a fee. Request permissions from permissions@acm.org.

FCDL '19, June 2019, Urbana-Champaign, Illinois

(c) 2019 Association for Computing Machinery.
}

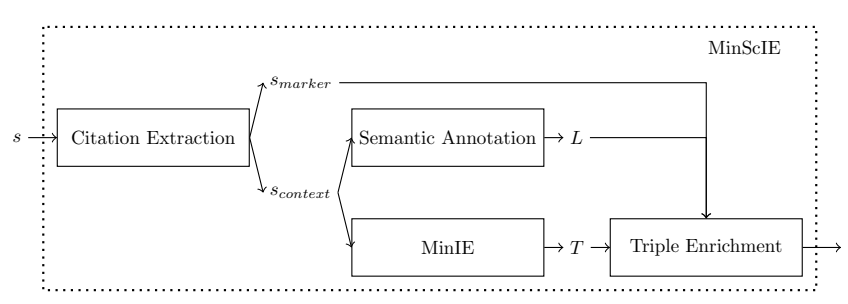

Figure 1: MinScIE's pipeline.

an extension of the OIE system MinIE [2], which is specifically designed to handle citations and semantically enrich the obtained triples when applied to scientific content. MinScIE provides fixes for most of the issues identified in [4] and further analyzes citation contexts regarding their citation polarity [1] and purpose [7]. We make MinScIE publicly available ${ }^{2}$ and provide an initial evaluation and analysis of the system's performance.

\section{SYSTEM DESCRIPTION}

MinScIE's pipeline approach is illustrated in Figure 1. Given a sentence $s=\left[w_{1}, \ldots, w_{n}\right]$ of length $n$ with individual tokens $w_{i}$, the system first extracts the citation marker, a subsequence of tokens $s_{\text {marker }}=\left[w_{j}, \ldots, w_{k}\right]$, with $k, j$ such that $k-j>0$ by matching the text to a regular expression. Next, we feed the remaining sequence $s_{\text {context }}$, which we consider the citation context, a) through a classifier for each of the respective annotation tasks, i.e., the citation polarity or the citation purpose models, and b) into the original MinIE core. Following Lauscher et al. [6], the system currently employs two Support Vector Machines (SVMs) with RBF kernel, for which we represent the context with sentence-averaged 300dimensional GloVe embeddings trained on computational linguistic publications . Note that the classification models are interchangable and that MinScIE can be easily adapted to handle a bigger set of scitorics [5]. As a result of the preceding steps, we obtain two labels $L$ reflecting the semantics of the citation based on the context provided by the surrounding sentence as well as a set of extracted triples $T=\left\{t_{1}, \ldots, t_{m}\right\}$ of size $m$. In a last step, we enrich the triples by creating a new MinIE triple annotation object consisting of the citation marker and the citation polarity and purpose labels identified, and adding it to each $t_{i} \in T$. The citation can be uniquely identified with the citation marker, which allows for citation-centered structured knowledge analysis in downstream applications.

$\overline{{ }^{2} \text { https://github.com/gkiril/MinSCIE }}$ 
Table 1: Evaluation results on 220 sentences from different scientific domains.

\begin{tabular}{lccccc}
\hline \multirow{2}{*}{ System } & \multirow{2}{*}{ \# Triples } & \multicolumn{3}{c}{ Correct } & \multicolumn{2}{c}{ Incorrect } \\
& & $\#$ & $\%$ & $\#$ & $\%$ \\
\hline MinIE & 846 & 568 & 67.14 & 278 & 32.86 \\
MinScIE & 822 & 577 & 70.19 & 245 & 29.81 \\
\hline
\end{tabular}

\section{EVALUATION}

For a detailed evaluation of the SVM model performances, we refer to Lauscher et al. [6]. Here, we focus on the evaluation of the quality of the extracted triples.

Data Set. For evaluating the triple extraction quality of our system, we use the SCI data set created by Groth et al. [4]. ${ }^{3}$ It consists of 220 sentences which were randomly sampled from 11 scientific articles covering different scientific domains, e.g. medicine, agriculture, and computer science. Before conducting the annotation process, we applied the original MinIE distribution in the safe mode as well as MinScIE to the each of the sentences in the SCI data set.

Annotation Process. In the annotation process, we evaluated the extracted triples from each of the two systems regarding their correctness. To this end, we hired three annotators and instructed them to follow the annotation guidelines created by Gashteovski et al. [2]. ${ }^{4}$ We split the data for both systems evenly between the annotators. Gashteovski et al. [2] report a moderate inter-annotator agreement of $50 \%-53 \%$ Cohen's $\kappa$ for untrained labelers.

Results. Table 1 shows the results of our evaluation. Overall, for 26 sentences (11.89\%) the processing between MinIE and MinScIE differed. As a result, MinScIE extracted 24 triples less than the original system and improved the performance by around $3 \%$ precision. Removing the citation markers before extracting the triples avoids confusing the core of our system, MinIE, in many cases and leads to better extractions. As an example, consider the following sentence:

"Some use strong extractants which dissolved strongly bound P and hence does not necessarily represent the actual labile pool of $P$ in soils and others use weak extractants like water or weak acids which might underestimate available soil P (Neyroud and Lischer, 2003)."

The original version of MinIE outputs the following triples:

$t_{1}$ : (water; is; weak extractant)

$t_{2}$ : (weak acids; is; weak extractant)

$t_{3}$ : (QUANT_S_1; use; strong extractants); QUANT_S_1 = some

$t_{4}$ : (strong extractants; dissolved; strongly bound $P$ )

$t_{5}$ : (strong extractants; does represent; actual labile pool of $P$ in soils)

$t_{6}:$ (others; use weak extractants like; water)

$t_{7}$ : (others; use weak extractants like; weak acids)

$t_{8}$ : (others; use; weak extractants)

$t_{9}:($ water; underestimate; available soil $P)$

$t_{10}$ : (weak acids; underestimate; available soil $P$ )

$t_{11}$ : (available soil $P$; is; Neyroud)

$t_{12}$ : (available soil $P$; is; Lischer)

\footnotetext{
${ }^{3}$ https://data.mendeley.com/datasets/6m5dyx4b58/2/files/f187c790-5770-408a-b04eba849d7d0261

${ }^{4}$ https://dws.informatik.uni-mannheim.de/fileadmin/lehrstuehle/pi1/pi1/minie/minielabeling-guide.pdf
}

In this example, the triples $t_{1}$ to $t_{10}$ are correctly extracted, but the last two triples, $t_{11}$ and $t_{12}$ are incorrectly extracted. The reason for this issue is that MinIE (which is based on dependency parsing output) cannot deal with citation markers, because the dependency parser confuses the words from within brackets from the citation markers as having appositional relationship with the head noun of the preceding noun phrase. In contrast, MinScIE extracts the same triples $t_{1}$ to $t_{10}$, but omits outputting the wrong triples $t_{11}$ and $t_{12}$. In addition, MinScIE extracts and preserves the citation marker, semantically analyzes the citation context, and attaches the obtained information to each of the triples:

\section{Citation Marker: (Neyroud and Lischer, 2003) \\ Citation Polarity: Neutral}

Citation Function: Criticizing

However, some issues identified in [4] are not resolved by applying this rather simple extension, e.g., the correct extraction of triples from sentences including complex mathematical formula.

\section{CONCLUSION}

In this work we presented MinScIE, an OIE system adapted to the scientific domain. By accounting for the occurance of citation markers in the text, the system offers a higher precision than it's nonadapted core, MinIE, and additionally provides a semantic analysis of the context of a citation in terms of citation polarity and purpose. We strongly believe that citation-centered OIE triples are useful representations of the knowledge inherent to scientific literature as they enable the user to connect factual knowledge with references to the scientific discourse. We also think that our technique for avoiding confusion in the triple extraction can be easily extended to other sources of confusion, e.g. intra-paper references to figures and tables. In the future, we plan to extend MinScIE for handling these cases and further enrich the semantic analysis component with more scitorics [5], e.g., argumentative information.

\section{REFERENCES}

[1] Awais Athar. 2011. Sentiment Analysis of Citations using Sentence StructureBased Features. In The 49th Annual Meeting of the Association for Computational Linguistics: Human Language Technologies, Proceedings of the Conference, 19-24 June, 2011, Portland, Oregon, USA - Student Session. 81-87. http://aclweb.org/ anthology/P11-3015

[2] Kiril Gashteovski, Rainer Gemulla, and Luciano Del Corro. 2017. MinIE: Minimizing Facts in Open Information Extraction. In Proceedings of the 2017 Conference on Empirical Methods in Natural Language Processing, EMNLP 2017, Copenhagen, Denmark, September 9-11, 2017. 2630-2640. http://aclweb.org/anthology/D17-1278

[3] G Nigel Gilbert. 1977. Referencing as persuasion. Social Studies of Science 7, 1 (1977), 113-122.

[4] Paul T. Groth, Michael Lauruhn, Antony Scerri, and Ron Daniel. 2018. Open Information Extraction on Scientific Text: An Evaluation. In Proceedings of the 27th International Conference on Computational Linguistics, COLING 2018, Santa Fe, New Mexico, USA, August 20-26, 2018. 3414-3423. http://aclweb.org/anthology/ C18-1289

[5] Anne Lauscher, Goran Glavaš, and Kai Eckert. 2018. ArguminSci: A Tool for Analyzing Argumentation and Rhetorical Aspects in Scientific Writing. In Proceedings of the 5th Workshop on Argument Mining, ArgMining@EMNLP 2018, Brussels, Belgium, November 1, 2018. 22-28. http://aclweb.org/anthology/W18-5203

[6] Anne Lauscher, Goran Glavaš, Simone Paolo Ponzetto, and Kai Eckert. 2017. Investigating Convolutional Networks and Domain-Specific Embeddings for Semantic Classification of Citations. In Proceedings of the 6th International Workshop on Mining Scientific Publications. Association for Computing Machinery, Toronto, ON, Canada, 24-28.

[7] Simone Teufel, Advaith Siddharthan, and Dan Tidhar. 2006. Automatic Classification of Citation Function. In Proceedings of the 2006 Conference on Empirical Methods in Natural Language Processing. Association for Computational Linguistics, Sydney, Australia, 103-110. 